\title{
Growth and Characterization of Bisthiourea Nickel Bromide-New Semiorganic Nonlinear Optical Crystal
}

\author{
R. S. Sundararajan, M. Senthilkumar, C. Ramachandraraja* \\ Department of Physics, Government Arts College (Autonomous), Kumbakonam, India \\ Email: *crraja_phy@yahoo.com
}

Received January $21^{\text {st }}, 2013$; revised February $25^{\text {th }}, 2013$; accepted March $3^{\text {rd }}, 2013$

Copyright (C) 2013 R. S. Sundararajan et al. This is an open access article distributed under the Creative Commons Attribution License, which permits unrestricted use, distribution, and reproduction in any medium, provided the original work is properly cited.

\begin{abstract}
A new semiorganic nonlinear optical crystal of bisthiourea nickel bromide (BTNB) was grown successfully by slow evaporation technique using water as solvent. The lattice parameters of the grown crystal have been determined by $\mathrm{X}$-ray diffraction studies. Vibrational spectrum is recorded to determine symmetries of molecular vibrations. Optical absorbance spectrum recorded revealed that this crystal has good transparency in the visible region. The second harmonic generation test of BTNB confirmed the nonlinear nature of the crystal. The BTNB crystal was analyzed by a differential thermal analysis and thermogravimetric analysis (DTA-TGA) to obtain its thermal stability. Vickers microhardness test done on the crystal shows that the crystal has greater physical strength.
\end{abstract}

Keywords: BTNB—Bisthiourea Nickel Bromide; SHG—Second Harmonic Generation; TGA/DTA; NLO-Nonlinear Optical

\section{Introduction}

Nonlinear optical (NLO) frequency conversion materials have a significant impact on laser technology, optical communication and optical storage technology. The search for new frequency conversion materials over the past decade has led to the discovery of many semiorganic NLO materials with high nonlinear susceptibilities. Among the semiorganic NLO materials metal complexes of the thiourea having lower cut off wavelengths, applicable for frequency conversion are of interest because both organic and inorganic components in it contribute specifically to the process of SHG [1-7]. In the present work metal replacement is studied by taking a reported NLO crystal bisthiourea cadmium bromide [8]. Here the metal cadmium is replaced by nickel which is more active electro chemically.

\section{Experimental}

High purity salts $(99.9 \%)$ purchased from E-Merck were used for the crystal growth experiments. Single crystals of bisthiourea nickel bromide (BTNB) were grown at room temperature by slow evaporation of on aqueous solution containing thiourea and nickel bromide in stoichiometric ratio $2: 1$ as per the reaction.

${ }^{*}$ Corresponding author.

$$
2 \mathrm{CS}\left[\mathrm{NH}_{2}\right]_{2}+\mathrm{NiBr}_{2} \rightarrow \mathrm{Ni}\left[\mathrm{CS}\left(\mathrm{NH}_{2}\right)_{2}\right]_{2} \mathrm{Br}_{2}
$$

Colouless crystals were harvested in about 110 days and are shown in Figure 1.

\section{Characterization}

Single crystal XRD analysis was carried out using on Enraf Nonius CAD-4. X-ray diffractometer with $M_{0} K_{\alpha}(\lambda$ $=0.770 \AA)$ radiation to identify the structure and to

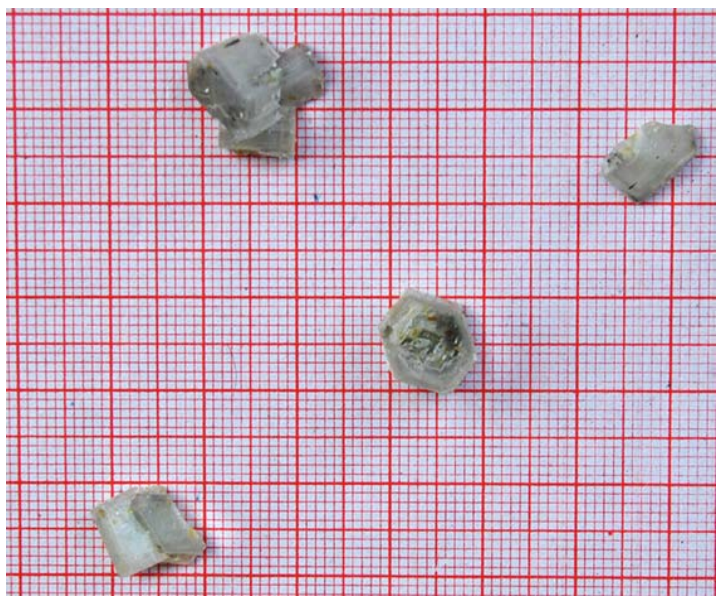

Figure 1. Photograph of the grown BTNB crystals. 
presence of functional groups in BTNB qualitatively, estimate lattice parameter values. In order to analyze the Fourier transform of infrared (FT-IR) spectrum was recorded in the range $400-4000 \mathrm{~cm}^{-1}$ using Perkin Elmer grating infrared spectrometer. The sample was in a pellet form in $\mathrm{KBr}$ phase. Linear optical properties of the crystals were studied using Varion Carey 5E UV-Vis-NIR spectrophotometer. Thermal studies were carried out using simultaneous thermogravimetric analyser (STA) 409C [NETZSCH.]. The nonlinear property of BTNB crystals was confirmed from second harmonic generation test by using Nd:YAG laser beam. The physical strength of the crystal was measured by Vicker's micro hardness test.

\section{Results and Discussion}

\subsection{X-Ray Diffraction Studies}

The single crystals of BTNB have been subjected to $\mathrm{X}$-ray diffraction studies using an ENRAF NONIUS CAD4 X-ray diffractometer to determine the cell structure and cell parameters. The studies revealed that the crystal belongs to orthorhombic system. In order to understand the role of metal ions in the grown crystals, a comparison is made between free ligand thiourea, bisthiourea cadmium bromide and bisthiourea nickel bromide and is given in Table $\mathbf{1}$.

\subsection{Analysis of IR Spectrum of Thiourea Vibrations in BTNB}

The IR spectrum of the free ligand and its transition metal complex bisthiourea cadmium bromide (BTCB) was reported earlier [8]. In the present study an attempt has been made to reveal the effect of coordination on the vibrational bands of the ligand, when Nickel replaces Cadmium. In the FT-IR spectrum (Figure 2) of BTNB, the $\mathrm{NH}$ stretching vibrational bands of $\mathrm{NH}_{2}$ were observed at 3376 and $3268 \mathrm{~cm}^{-1}$. The $\mathrm{NH}_{2}$ bending vibration which was observed at $1614 \mathrm{~cm}^{-1}$ for BTCB is found at 1601 $\mathrm{cm}^{-1}$ for BTNB. In BTCB the C-S stretching vibration was found at $1434 \mathrm{~cm}^{-1}$ whereas it was recorded at 1410 $\mathrm{cm}^{-1}$ for BTNB. The IR band for $\mathrm{NH}_{2}$ rocking was observed at $1096 \mathrm{~cm}^{-1}$ for BTCB is recorded at $1080 \mathrm{~cm}^{-1}$ for BTNB. The symmetric stretching band is recorded at $716 \mathrm{~cm}^{-1}$ and $726 \mathrm{~cm}^{-1}$ for BTCB and BTNB respectively. The IR band of N-C-S bending vibration is observed at $555 \mathrm{~cm}^{-1}$ for BTCB whereas it is observed at $494 \mathrm{~cm}^{-1}$ for BTNB. This study (Table 2) reveals that when nickel replaces cadmium, the corresponding vibrational frequencies are greatly altered.

\subsection{Optical Transmission Spectrum}

Optical absorption spectrum (Figure 3) of BTNB was

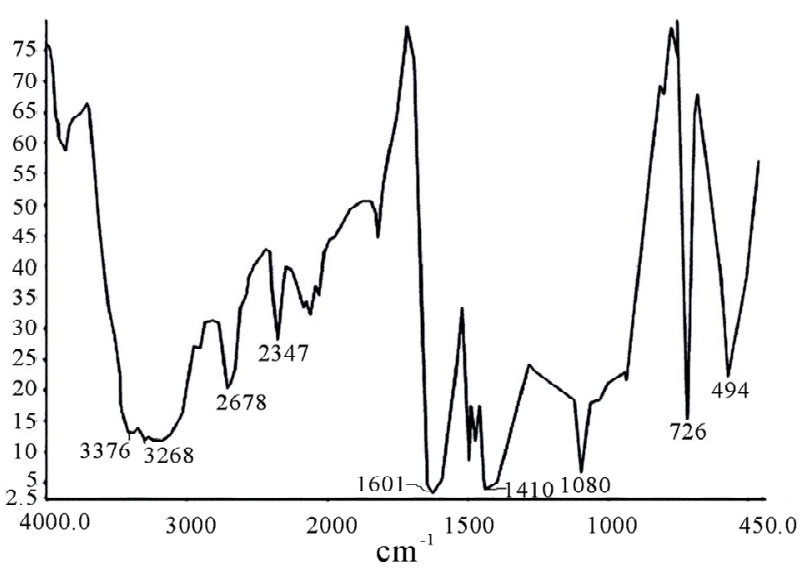

Figure 2. FT-IR Spectrum of the grown BTNB crystals.

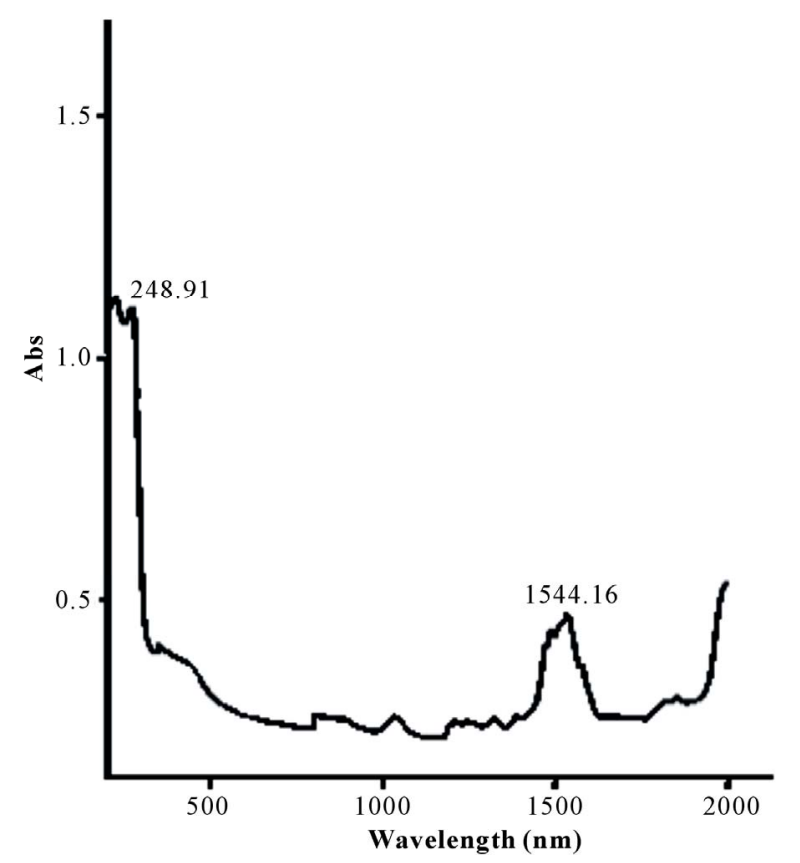

Figure 3. Absorbance spectrum of the grown BTNB crystals.

recorded by employing Varion Carey 5E UV-Vis-NIR spectrophotometer in the range $200-2000 \mathrm{~nm}$. The lower cut off for BTNB is observed at $248.91 \mathrm{~nm}$ whereas it was observed at $269 \mathrm{~nm}$ for BTCB. The shifting of lower cut off in UV region further recommends the crystal for optoelectronic applications. The crystal has sufficient transmission in the entire visible region.

\subsection{Second Harmonic Generation}

The SHG of BTNB single crystal was studied using $\mathrm{Nd}$ :YAG laser (model continuum YG501C, $\lambda=1064 \mathrm{~nm}$ ) by Kurtz-Perry technique [9]. Powdered sample of the investigated crystal was taken in a glass capillary tube. It was irradiated by the laser pulse and second harmonic signal was detected using an optical cable attached to 
Table 1.

\begin{tabular}{ccccccccc}
\hline S.No & Sample & $\mathrm{a}(\AA)$ & $\mathrm{b}(\AA)$ & $\mathrm{c}(\AA)$ & $\mathrm{A}\left({ }^{\circ}\right)$ & $\mathrm{B}\left({ }^{\circ}\right)$ & $\Gamma\left(^{\circ}\right)$ & Volume $\left(\AA^{3}\right)$ \\
\hline 1 & Thiourea & 7.655 & 8.537 & 5.520 & 90 & 90 & 90 & 360.732 \\
2 & BTCB & 5.6949 & 6.6051 & 7.5937 & 90 & 90 & 90 & 285.639 \\
3 & BTNB & 6.1310 & 8.0907 & 9.1131 & 90 & 90 & 90 & 352.838 \\
\hline
\end{tabular}

Table 2.

\begin{tabular}{cccc}
\hline IR TU m$^{-1}$ & ${\text { BTCB } \mathbf{~ c m}^{-1}}^{-1}$ & ${\text { BTNB } \mathbf{~ c m}^{-1}}^{-1}$ & Assignment \\
\hline 3380 & - & 3376 & $v(\mathrm{~N}-\mathrm{H})$ \\
3279 & 3297 & 3268 & $v(\mathrm{~N}-\mathrm{H})$ \\
1620 & 1614 & 1601 & $\delta(\mathrm{N}-\mathrm{H})$ \\
1477 & 1468 & - & $v(\mathrm{~N}-\mathrm{C}-\mathrm{N})$ \\
1414 & 1434 & 1410 & $v(\mathrm{C}-\mathrm{S})$ \\
1082 & 1096 & 1080 & $\rho \mathrm{NH}_{2}$ \\
730 & 716 & 726 & $v(\mathrm{C}-\mathrm{N})$ \\
494 & 555 & 494 & $\delta(\mathrm{N}-\mathrm{C}-\mathrm{S})$ \\
\hline
\end{tabular}

$v$-Stretching; $\rho$-rocking; $\delta$-deformation.

fluorescence spectroscope (Princeton instrument Int. Spectroscopy instrument GmbH, model DIDA-512 GIR). The SHG output of the BTNB crystal was found to be 68 $\mathrm{mV}$ which is lesser when compared with SHG output of BTCB $(98 \mathrm{mV})$ for laser beam of same intensity. The SHG output confirms the nonlinear nature of the experimental crystal.

\subsection{TGA/DTA-Analysis}

The thermo gravimetric analysis and differential thermal analysis (TGA and DTA) curves for BTNB were obtained by using simultaneous thermo gravimetric analyser (STA) $409 \mathrm{C}(\mathrm{NETZSCH})$ at a heating rate of $10^{\circ} \mathrm{C} /$ min in nitrogen atmosphere and are reported in Figure 4. The TGA curve shows that there was a weight loss of about $76 \%$ in the temperature range $182^{\circ} \mathrm{C}-607^{\circ} \mathrm{C}$.

The first endothermic peak at $189.08^{\circ} \mathrm{C}$ in the DTA curve may be due to the liberation of bromide and the second endothermic peak at $239.18^{\circ} \mathrm{C}$ may be due to liberation of thiourea in BTNB. The loosely bound nature of bromide in the grown crystal is confirmed by the formation of pale yellow precipitate which is sparingly soluble in ammonia when the powdered sample of the grown crystal is treated with silver nitrate in acid medium.

\subsection{Microhardness Studies}

Vickar's microhardness test conducted on the experimental crystal proves its greater physical strength. It is well explained by the increase in hardness value with increase in Load (Table 3).

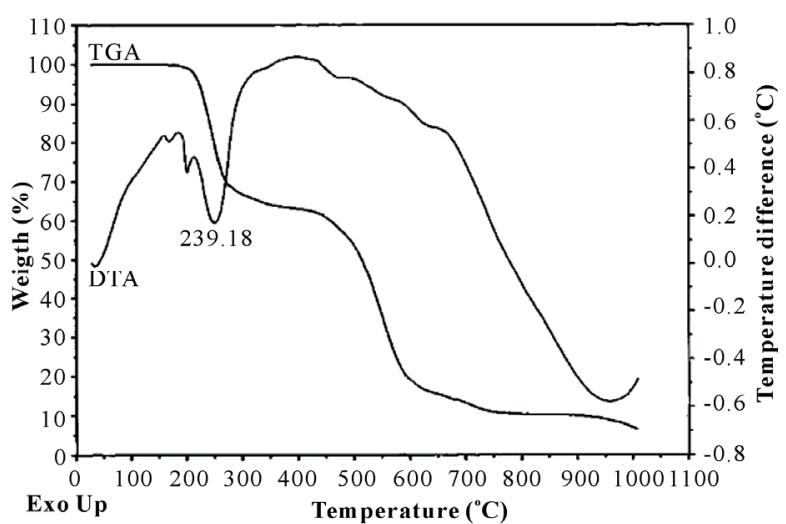

Figure 4. TGA/DTA curves of the grown BTNB crystals.

Table 3.

\begin{tabular}{cc}
\hline Load grams & BTNB HV \\
\hline 25 & 39.3 \\
50 & 52.4 \\
100 & 71.7 \\
\hline
\end{tabular}

\section{Conclusion}

Single crystals of BTNB a new semi organic NLO martial have been grown from aqueous solution. A detailed comparison of vibrational data of BTNB with BTCB reveals that when nickel replaces cadmium, they are greatly altered. The optical absorption spectrum shows that lower cut off for BTNB is smaller than that of BTCB. The SHG output confirms the nonlinear nature of the crystal. The TGA/DTA curves and Vicker's microhardness test confirms the thermal stability and physical strength of the investigated crystal.

\section{Acknowledgements}

The authors are thankful to Madurai Kamaraj University, Madurai, CECRI Karaikudi, St. Joseph's College, Trichy and I.I.Sc, Bangalore for the spectral facilities.

\section{REFERENCES}

[1] V. Krishnakumar and R. Nagalakshmi, "Crystal Growth and Characterization of $\mathrm{K}\left[\mathrm{CS}\left(\mathrm{NH}_{2}\right)_{2}\right]_{4} \mathrm{Br}-\mathrm{A}$ Semiorganic Non-Linear Optical Crystal," Spectrochimica Acta Part A: Molecular and Biomolecular Spectroscopy, Vol. 68, No. 3, 2007, pp. 443-453. 
doi:10.1016/j.saa.2006.11.049

[2] N. Vijayan, R. Ramesh Babu, et al., "Some Studies on the Growth and Characterization of Organic Nonlinear Optical Acetoacetanilide Single Crystals," Journal of Crystal Growth, Vol. 267, No. 3-4, 2004, pp. 646-653. doi:10.1016/j.jcrysgro.2004.04.008

[3] R. Mohankumar, D. Rajanbabu, et al., "Studies on the Growth Aspect of Semiorganic 1-Alanine Acetate a Promising Nonlinear Optical Crystal," Journal of Crystal Growth, Vol. 275, No. 1-2, 2005, p. e1935-e1939. doi:10.1016/j.jcrysgro.2004.11.260

[4] H. O. Merry, L. F. Warren, et al., "Liquid Level Sensor with Optical Fibers," Applied Optics, Vol. 31, 1992, p. 5051.

[5] V. Venkataraman, G. Dhavaraj, H. L. Bhat, et al., "Crystal Growth and Defect Characterization of Zinc Tris(thiourea) Sulfate: A Novel Metal-Organic Nonlinear Optical Crystal," Journal of Crystal Growth, Vol. 154, No. 1-2, 1995, p. 92-97. doi:10.1016/0022-0248(95)00212-X
[6] V. Venkataraman, S. Maheswaran, et al., "Crystal Growth and Physical Characterization of the Semiorganic Bis(thiourea) Cadmium Chloride Crystals," Journal of Crystal Growth, Vol. 174, No. 3-4, 1997, p. 605-610. doi:10.1016/S0022-0248(97)00137-1

[7] D. Xu, M. Jiaog and Z. Taus, "A New Phase Matchable NLO Crystal 1-Arginine Monohydrate," Acta Chimica Sinica, Vol. 41, 1983, p. 570

[8] C. Ramachandraraja and R. S. Sundararajan, "Growth, Characterization, Vibrational Spectroscopic and Thermal Studies of a New Metallorganic Non-Linear Optical Crystal-Bisthiourea Cadmium Bromide," Spectrochimica Acta Part A: Molecular and Biomolecular Spectroscopy, Vol. 71, No. 4, 2008, pp. 1286-1289. doi:10.1016/j.saa.2008.03.028

[9] S. K. Kurtz and T. T. Perry, "A Powder Technique for the Evaluation of Nonlinear Optical Materials," Journal of Applied Physics, Vol. 39, No. 8, 1968, p. 3798-3814. doi:10.1063/1.1656857 